\title{
Spectral analysis of cortical EEG activity during visual attention
}

\author{
LYNN C. OATMAN \\ U.S. Army Human Engineering Laboratory, Aberdeen Proving Ground, Maryland
}

\begin{abstract}
Cortical EEG activity was recorded from four chronically implanted cats before, during, and after the presentation of a visual discrimination task. Sample epochs of auditory cortex EEG activity from the three attentive states, in the absence of bodily movement, were subjected to the fast Fourier transform, and the average power spectra were determined for the frequency bands theta $(4-8 \mathrm{~Hz})$, alpha $(8-13 \mathrm{~Hz})$, beta $1(13-20 \mathrm{~Hz})$, and beta $2(20-40 \mathrm{~Hz})$. The amount of power within the theta frequency range was significantly larger when the attention of the cats was focused on the visual discrimination than when they were nonattentive. Increased visual attention had no systematic effect on the amount of power occurring in the alpha, beta 1 , and beta 2 frequency bands. The results suggest that the function of the hippocampus in selective attention is one of gating or filtering, which allows an animal to ignore or filter out background, irrelevant stimuli.
\end{abstract}

In recent years, evidence has accumulated that supports the idea that the hippocampus, and specifically the hippocampal theta waves or RSA (rhythmic slow activity), plays a role in the mechanism that allows an organism to process sensory information selectively. Several studies have shown a correlation between hippocampal electrical activity and attention (Adey, 1967; Bennett, 1970, 1971, 1975; Coleman \& Lindsley, 1975; Kemp \& Kaada, 1975). Douglas $(1967,1972)$ suggested that the hippocampus functions as a sensory gating center. It is postulated that, during selective attention, the hippocampus excludes irrelevant stimulus patterns by inhibitory modulation of sensory input. Other investigators (Moore, 1979; Solomon, 1979, 1980; Solomon \& Moore, 1975) have also suggested that the hippocampus is involved in inhibiting or tuning out irrelevant stimuli.

In previous studies, Oatman $(1971,1976)$ suggested that, during attention to visual stimulation, a central inhibitory mechanism suppresses irrelevant auditory stimuli through the action of the olivocochlear bundle (OCB). In animals exhibiting various behaviors, the OCB has been shown to inhibit auditory input at the receptor level during habituation and distraction experiments (Buño, Velluti, Handler, \& Garcia-Austt, 1966) and during attentive behavior to a visual stimulus (Oatman, 1971, 1976; Oatman \&

In conducting the research described herein, the investigator adhered to the Guide for Laboratory Animal Facilities for Laboratory Animal Resources, National Academy of Sciences, National Research Council, Washington, D.C. This paper is part of the public domain and may be reproduced in full or in part for any purpose of the U.S. Government. The author gratefully acknowledges the assistance of Donna M. Whitacre in reducing the data. The author's mailing address is: U.S. Army Human Engineering Laboratory, Aberdeen Proving Ground, Maryland 21005.
Anderson, 1977). This evidence has led to the belief that the OCB performs an inhibitory function by controlling auditory input to the central nervous system (CNS) at the peripheral level. The question remains as to whether the hippocampus performs an inhibitory function in experiments in which the OCB has been shown to inhibit auditory input to the CNS at the peripheral level.

The purpose of this experiment was to study the relationships between the electroencephalographic activity (EEG) of the CNS and attentive behavior. A visual discrimination task using instrumental conditioning was established in each animal in order to investigate EEG electrical patterns associated with different attentive states of the animal. The study was designed to determine if hippocampal theta waves recorded from the auditory cortex are present while an animal is attending to visual stimulation. Although theta waves are most prominent in the hippocampus, they are readily recorded from many regions of the brain, including the auditory cortex (Grastyán, Lissák, Madarász, \& Donhoffer, 1959; Green \& Arduini, 1954; McFarland, Teitelbaum, \& Hedges, 1975). It was thought that, if the hippocampus functioned to filter or inhibit irrelevant information as Douglas $(1967,1972)$ had proposed, then hippocampal activity (theta waves) would be increased during attention to a visual discrimination task, when the OCB has been shown to suppress irrelevant auditory input at the receptor level in the unanesthetized, unrestrained animal.

\section{METHOD}

\section{Subjects}

Four female cats with electrodes surgically placed at the auditory cortex served as subjects for this experiment. The cor- 
tical electrodes were flattened monopolar silver-ball electrodes stereotaxically implanted on the dura over the auditory cortex (AI). The indifferent electrode was a stainless steel screw placed over the frontal sinus. A detailed explanation of the surgical preparation used in this experiment can be found elsewhere (Oatman, 1971, 1976).

\section{Histology}

At the end of the experiment, the cats were given a lethal overdose of sodium pentobarbital administered intravenously. The brains were removed and placed in Formalin and potassium ferrocyanide for $24 \mathrm{~h}$. Examination of the brains confirmed that the auditory electrodes were placed in the middle ectosylvian gyrus (AI).

\section{Visual and Acoustic Stimulation}

While a detailed explanation of the methods employed in this experiment can be found elsewhere (Oatman, 1971, 1976), a brief summary follows. The visual stimuli consisted of two concentric rings presented successively for discrimination. The large outer ring was presented first, which served as a warning stimulus for the cat to attend to the stimuli. Then the smaller inner ring was presented. To receive a food reward, the cat had to push a response button on the floor of the test box when the small inner ring was presented. Figure 1 shows a schematic diagram of the presentation of the stimuli. If the cat responded between the presentation of the large outer ring and the presentation of the small inner ring, it received no reinforcement and the onset of the next trial was delayed $25 \mathrm{sec}$. To increase the cat's attentiveness and to avoid temporal conditioning, the time interval (T1) between the presentations of the large and the small concentric rings was varied randomly between 1 and $6 \mathrm{sec}$. The exposure duration of the small concentric ring was $4 \mathrm{sec}$, and the time between trials was varied from 20 to 45 sec.

Auditory tone bursts were presented continuously at a rate of $1 / \mathrm{sec}$ during the presentation of the successive visual discrimination task, but they were not synchronized with the onset of the visual display. The tone bursts were presented through a soundtube system at an intensity of $85 \mathrm{~dB}$ SPL (re $.0002 \mu \mathrm{bar}$ ) at a rate of $1 / \mathrm{sec}$ at each of the 10 frequencies ranging from $200 \mathrm{~Hz}$ to $10 \mathrm{kHz}$. This intensity ( $85 \mathrm{~dB})$ was selected because the cochlear microphonics were in the linear portion of its input-output function. Tone bursts were presented through a sound-tube system which terminated at the entrance to the cat's external meatus. The sound tube was not fastened to the pinna but was held firmly in place by a bracket attached to the electrode plug. Sound pressures were calibrated with a $.635-\mathrm{cm}$ condensor microphone (Brüel and Kjaer Type 4135) and placed perpendicularly to and just in front of the end of the sound tube. Movements of the sound tube to different positions within the test cubicle did not change the output voltage from the microphone.

\section{Data Collection and Procedure}

Four weeks after surgery, the cats were placed into the soundattenuating test cubicle and electrodes were checked. Simultaneous tone-burst-evoked responses were recorded from the audi-

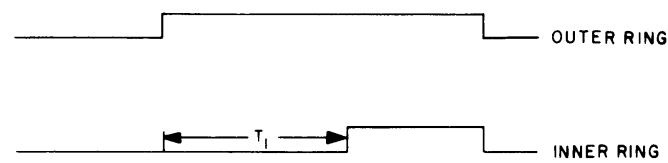

几 BEHAVIORAL RESPONSE

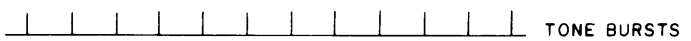

Figure 1. Schematic diagram of the presentation of the stimuli. tory cortex. These data were collected during recording sessions that consisted of three different periods designed to effectively manipulate the attentive state of the subjects: (1) a pretest control, in which the cat was awake, relaxed and not attentive to any identifiable stimuli, (2) an experimental period during the visual discrimination between the presentation of the outer and inner concentric rings (T1) when the cat was attentive, and (3) a posttest control period similar to the present control period. The evoked responses for each of the three attentive states were then collected at each of 10 different frequencies (1 frequency per day) in an order of presentation descending from $10 \mathrm{kHz}$ to $200 \mathrm{~Hz}$. The tone-burst-evoked response data from this experiment have been previously reported (Oatman \& Anderson, 1977).

\section{Spectral Analysis}

Spectral analysis of the auditory cortex EEG was performed offline by a Nicolet Med 80 computer for each of the three attentive states. The EEG signals from the auditory cortex were recorded on a Grass Model 7 polygraph (with $1-\mathrm{Hz}$ and $3-\mathrm{kHz}$ half-amplitude cutoff points) and a Sangamo Model 4700 FM tape recorder at a speed of $7 \frac{1}{2} \mathrm{ips}$ ). The EEG signals were filtered (Krohn Hite Model 3343) from $1 \mathrm{~Hz}$ to $50 \mathrm{~Hz}$ and fed into a Nicolet Med 80 computer, which performed a spectral analysis using a fast Fourier transform. Each of the EEG and computer inputs was calibrated with a $50-\mu v, 10-\mathrm{Hz}$ signal before each run. For each attentive state, four epochs of $5.12 \mathrm{sec}$ of artifact-free EEG data were taken. Movement artifacts were automatically removed by the artifactreject mode of the computer. In this mode, the computer acquires and examines each epoch of the data prior to accepting it. The epochs with gross movement artifacts containing abnormally large y-excursions are thrown out. The threshold for rejection was set using control data derived when the animal was quiet, relaxed, and not attentive, and then the threshold remained constant for all three attentive states.

Following the artifact removal, the Nicolet frequency analysis software transformed each successive 5.12-sec epoch. The four spectral transforms were then added together and printed out, as well as plotted by the computer as one line on a Hewlett-Packard (Model 7035B) x-y plotter (Figure 2). Figure 2 shows an example (derived from one of the cats) of a computer plot of 10 spectral transforms, each based on four 5.12-sec epochs. The power spectra were computed in the frequency range of $1-50 \mathrm{~Hz}$ and plotted between 4-40 $\mathrm{Hz}$, with a resolution of $1 \mathrm{~Hz}$. The power spectra were printed out into the frequency bands theta $(4-8 \mathrm{~Hz})$, alpha (8-13 Hz), beta $1(13-20 \mathrm{~Hz})$, and beta $2(20-40 \mathrm{~Hz})$ (Doyle, Ornstein, \& Galin, 1974; Gevins, Zeitlin, Yingling, Doyle, Dedon, Schaffer, Roumasset, \& Yeager, 1979). The average power spectra were based on 18 spectral transforms of four 5.12 -sec epochs, which yielded a total analysis of $6 \mathrm{~min} 14 \mathrm{sec}$ and provided an average power density spectrum for each of the three attentive states for each animal.

\section{RESULTS}

The relation between attentive behavior and cortical EEG activity to be described here is based on a power spectral analysis of the auditory cortex EEG signals. Representative power spectrum plots obtained from one of the animals is presented in Figure 2. This figure shows 10 spectral transforms, each based on an average of four 5.12-sec epochs, for one cat on the 1st day of recording. The spectral transforms are plotted as a function of frequency for each of the three attentive states: pretest-control period (cat nonattentive, relaxed, but awake), experimental period (during visual discrimination, cat very attentive), and posttest-control period (cat nonattentive, relaxed, but awake). 

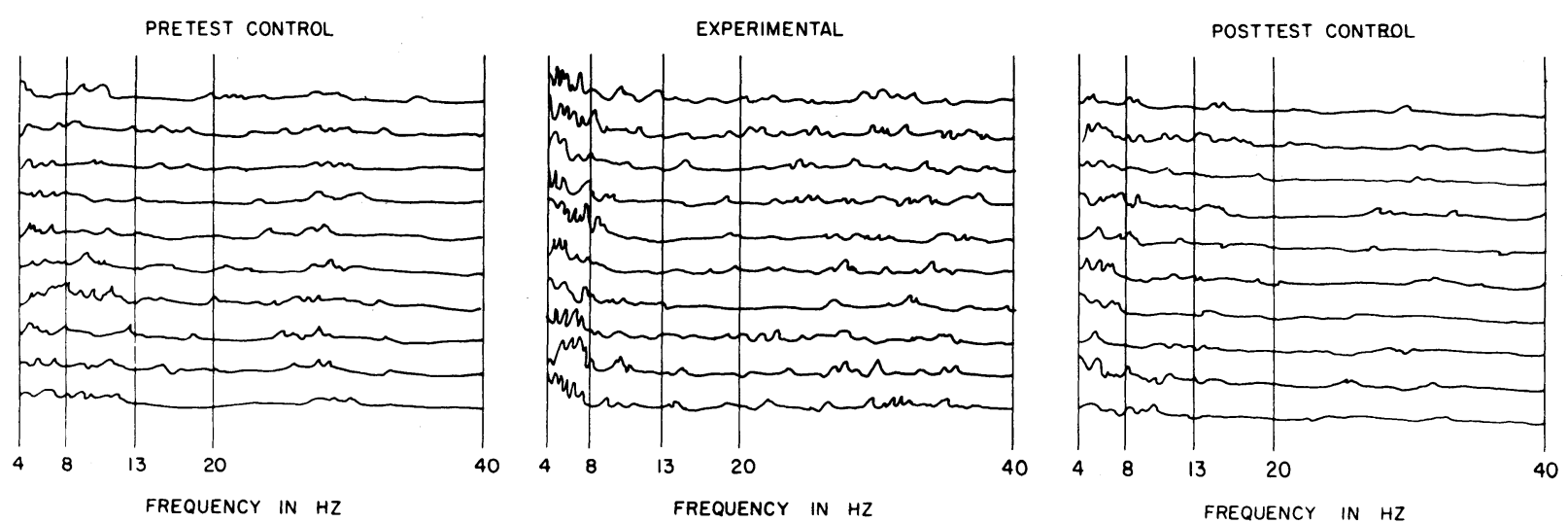

AUDITORY CORTEX

Figure 2. An example of a computer plot of 10 spectral transforms from the auditory cortex of one adult female cat as a function of attentive (experimental) and nonattentive (controls) states. Each line represents a power spectrum of four 5.12-sec epochs printed out in to the frequency bands theta $(4-8 \mathrm{~Hz})$, alpha $(8-13 \mathrm{~Hz})$, beta $1(13-20 \mathrm{~Hz})$, and beta $2(20-40 \mathrm{~Hz})$.

Figure 2 shows that when the attention of the animals was focused on the visual discrimination, greater activity occurred in the $4-8-\mathrm{Hz}$ (theta) band than when they were nonattentive. A trend toward greater power in the $20-40-\mathrm{Hz}$ (beta 2 ) band during visual attention was also noted.

In order to quantify these changes and provide a means to compare (using statistical measures) the EEG activity among the three attentive states, the mean power spectra was computed for the four frequency bands theta $(4-8 \mathrm{~Hz})$, alpha $(8-13 \mathrm{~Hz})$, beta 1 $(13-20 \mathrm{~Hz})$, and beta $2(20-40 \mathrm{~Hz})$ for 7 days of recording. In all, approximately 1,512 power-density spectra (18 spectral transforms $\times 3$ attentive states $\times 4$ animals $\times 7$ days) were computed, and the mean power-density spectra as a function of attentive state and frequency bands appear in Figure 3. Figure 3 shows for the 4-8- $\mathrm{Hz}$ (theta) band a significantly greater amount of power when the attention of the animals was focused on the visual discrimination than when they were nonattentive. An analysis of variance (Butler, Kamlet, \& Monty, 1969) indicated significant differences between the pretest-control period and the experimental period $[F(1,39)=28.05]$ and between the posttest-control period and the experimental period $[F(1,39)=14.50]$, but no significant differences between the pretest-control period and the posttest-control period $[F(1,39)=3.75]$. No significant differences were found among the three attentive states (pretest control, experimental, post-

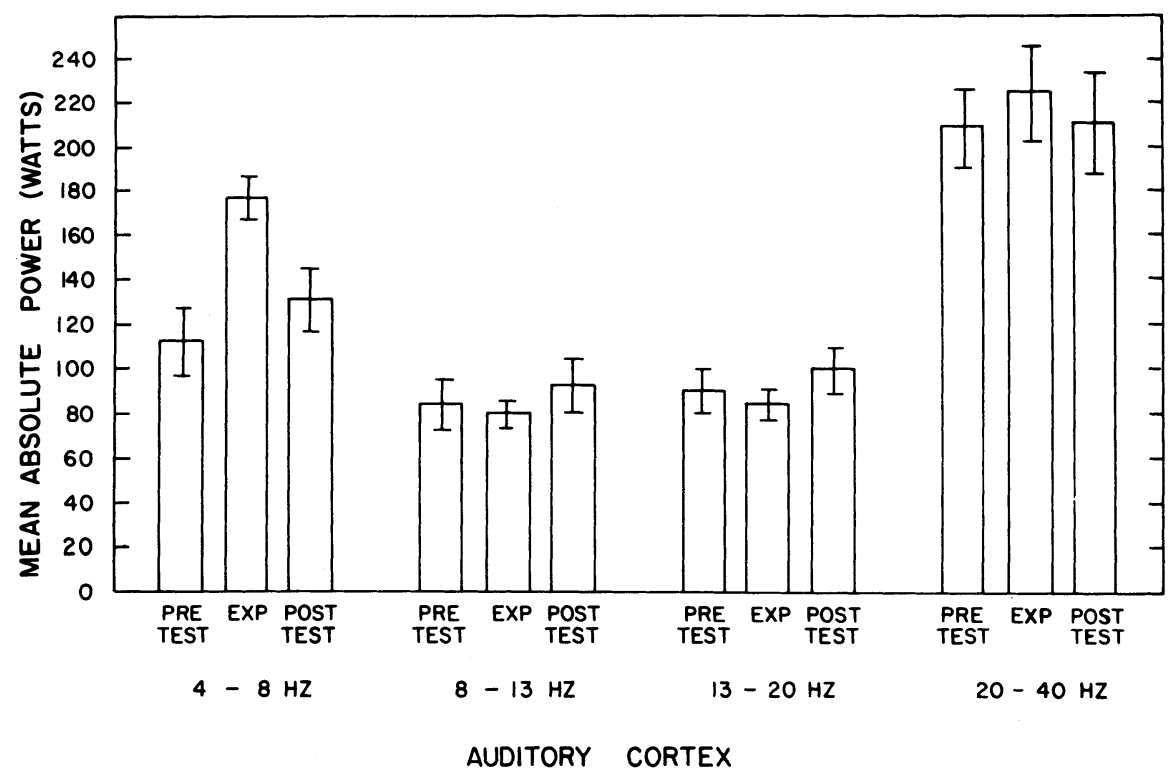

Figure 3. The mean absolute power computed for the four frequency bands as a function of attentive (exp) and nonattentive (pre- and posttest) states. The limits around each mean represent $\mathbf{9 5 \%}$ confidence levels. 
test control) in the 8-13-, 13-20-, and $20-40-\mathrm{Hz}$ bands.

Figure 3 also shows the greatest amount of power in the $20-40-\mathrm{Hz}$ band, with lower amounts of power occurring in the 4-8- $\mathrm{Hz}$ band and the least amount of power occurring in the 8-13- and $13-20-\mathrm{Hz}$ bands. An analysis of variance indicated significant differences when the $20-40-\mathrm{Hz}$ band was compared with the $4-8-\mathrm{Hz}$ band $[\mathrm{F}(1,123)=7.97]$, the $8-13-\mathrm{Hz}$ band $[F(1,123)=29.17]$, and the $13-20-\mathrm{Hz}$ band $[\mathrm{F}(1,123)$ $=32.81]$. The analysis also showed significant power differences between the $4-8-\mathrm{Hz}$ band and the $8-13-\mathrm{Hz}$ band $[\mathrm{F}(1,123)=27.12]$, as well as the $13-20-\mathrm{Hz}$ band $[F(1,123)=21.51]$. No significant differences in power were observed between the $8-13-\mathrm{Hz}$ band and the $13-20-\mathrm{Hz}$ band $(\mathrm{F}<1)$.

The mean power spectra of the cortical EEG activity as a function of attentive state and frequency band are plotted across 7 days in Figure 4. Figure 4 shows that the mean amount of power changed very little as a function of days for the three frequency bands 4-8 Hz, 8-13 Hz, and 13-20 Hz. An analysis of variance yielded no significant differences across the 7 days for the $4-8-\mathrm{Hz}$ band $[\mathrm{F}(6,39)=1.16], 8-13-\mathrm{Hz}$ band $(\mathrm{F}<1)$, and $13-20-\mathrm{Hz}$ band $(\mathrm{F}<1)$. However, in the $20-40-\mathrm{Hz}$ band, the amount of power was significantly $[F(6,39)=5.31]$ reduced as the number of days increased, for all three attentive states. The figure also shows that when the attention of the animals was focused on the visual discrimination, mean power spectra in the 4-8- $\mathrm{Hz}$ band (theta) were larger for all 7 days than when they were nonattentive.

\section{DISCUSSION}

The main objective of this study was to correlate the patterns of EEG activity of the CNS with different attentive states. This experiment demonstrated a correlation between the attentive state of the animal and hippocampal theta activity recorded from the auditory cortex. The power (amount of energy) within the theta frequency range was significantly larger when the attention of the cats was focused on the visual discrimination than when they were nonattentive. When the cat was awake, relaxed, and nonattentive, the hippocampal electrical activity had an irregular pattern and the total power of theta was attenuated. In contrast, when the cat was alert and attentive to the visual discrimination, the hippocampal electrical activity was dominated by the theta rhythm and the total power of theta increased. These results are consistent with the previous findings of Coleman and Lindsley (1975, 1977), Elazar and Adey (1967), and Kemp and Kaada (1975), who observed a predominance of hippocampal theta activity in cats exhibiting behavioral states of alertness and attentiveness.

The present data, which reflect an increase in hippocampal activity (theta waves) during attention to a visual discrimination task, suggest that the concept of a central inhibitory process that modulates afferent sensory information involves the hippocampus. In addition, the increased hippocampal theta activity was present during attention to a visual discrimination task, when the OCB has been shown to

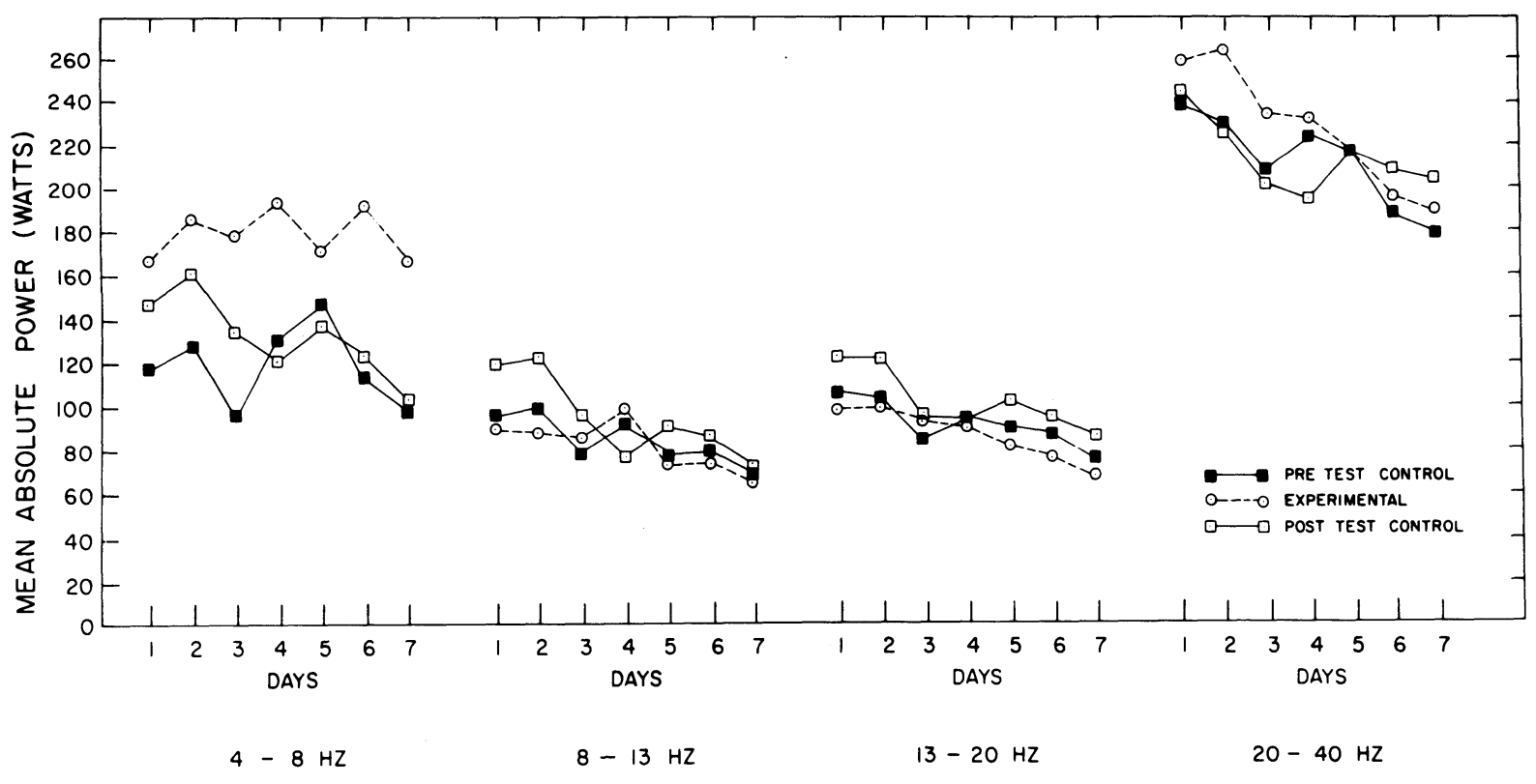

AUDITORY CORTEX

Figure 4. The mean absolute power computed for the four frequency bands as a function of days of recording and attentive (experimental) and nonattentive (controls) states. 
suppress irrelevant auditory input at the receptor level in the unanesthetized, unrestrained animal (Oatman \& Anderson, 1977). Apparently, the hippocampus is part of an inhibitory circuit that gates sensory stimuli through the OCB. The neural hippocampal output in response to irrelevant stimuli may act to inhibit or excite the brainstem reticular formation, which exerts both inhibitory and facilitatory influence at all levels of the specific sensory pathways (Hernández-Peón, 1961). Anatomical relationships between the hippocampus and the mesencephalic and diencephalic reticular formations and between the hypothalamus and the hippocampus have been described (Nauta, 1963). Electrical stimulation of the hippocampus can inhibit afferent input into the hypothalamus (Feldman, 1962) and can also result in the reduction of auditory-cortex evoked potentials (Parmeggiani \& Rapisarda, 1969; Redding, 1967). These results, together with our previous data on the auditory evoked potentials, support the theory suggested by Douglas $(1967,1972)$ that the function of the hippocampus in selective attention is one of gating or filtering, which allows the organism to ignore or filter out background, irrelevant stimuli. This view is in agreement with that of several other investigators (Moore, 1979; Solomon, 1979, 1980; Solomon \& Moore, 1975) who have presented evidence suggesting that the hippocampus is involved in inhibiting or tuning out irrelevant stimuli.

In contrast with the above experiments, several investigators (Arnolds, Lopes da Silva, Aitink, \& Kamp, 1979; Black, Young, \& Batenchuk, 1970; Robinson \& Vanderwolf, 1978; Vanderwolf, 1971) have proposed that, in some animals, hippocampal theta is a correlate of voluntary movement. Theta waves accompanied voluntary movements such as walking, running, climbing, head movements, etc., and the hippocampal theta was present as long as the movement continued, even up to $8 \mathrm{~h}$ (Whishaw \& Vanderwolf, 1971). Frederickson, Lenig, and Frederickson (1980) reported that movements per se were reliably accompanied by hippocampal theta activity. They also suggested that such a finding underscored the importance (first emphasized by Whishaw \& Vanderwolf, 1973) of controlling for voluntary movements in experiments investigating the correlates of hippocampal theta activity. The present experiment controlled for the occurrence of major skeletal movements by using the computer's artifactreject mode to automatically remove all bodily movements from the EEG data. In the artifact-reject mode, the computer acquired and examined each epoch of data before it would accept it. The epochs with gross bodily movements such as head movements, walking, barpressing, eating, licking, etc., were discarded from the analysis.

Despite the strong correlation between the hippocampal theta activity and voluntary movements, many investigators (Bennett, 1970, 1975; Coleman \& Lindsley, 1975; Frederickson et al., 1980) have argued that the hippocampal theta waves occur in the absence of bodily movement and are directly related to attention variables. In the present experiment, the results showed increased hippocampal theta activity during attentive behavior in the absence of bodily movement. This finding is in agreement with that of Coleman and Lindsley (1975), who have observed theta waves during immobile behavior states of alertness and attentiveness and have suggested that something other than voluntary motor activity is associated with theta waves. This suggestion is supported by Harper (1971), who found that hippocampal theta was present in the absence of movement in the rabbit and was present even during slow-wave sleep, and by Frederickson et al. (1980), who observed that an impending relevant sensory cue may directly influence the occurrence of hippocampal theta activity during immobility in cats. In addition, Buzsáki, Grastyán, Tveritskaya, and Czopf (1979) and Buzsáki, Haubenreiser, Grastyán, Czopf, and Kellényi (1981) observed that attention to a conditioned stimulus was accompanied by greater amounts of theta activity than were goal-directed voluntary behaviors, which suggested to those authors that hippocampal theta was not a correlate of motor activity.

Green and Arduini (1954) found that the occurrence of regular theta activity in the hippocampal EEG of the cat is generally accompanied by both neocortical and behavioral signs of arousal. In addition, Kemp and Kaada (1975), using pupillary size and amount of neocortical desynchronization as measures of arousal, have observed a clear positive relationship between moderate increases in level of arousal and amount of hippocampal theta activity. In light of these results, the role of arousal in this experiment must be clarified. In attention experiments, when an animal is conditioned to become attentive to a specific stimulus, the animal's general level of arousal might be increased simultaneously. Likewise, if the animal's arousal increased due to food reward during the visual discrimination task, then the increased theta activity might be due to nonspecific arousal rather than to selective attention. The analysis of the EEG activity recorded from the auditory cortex in the present experiment indicated that increased attention failed to produce a state of increased arousal. Increased visual attention had no systematic effect on the amount of power occurring in the 8-13- (alpha), 13-20- (beta 1), and 20-40-Hz (beta 2) bands. The present experiment seems to indicate that changes in nonspecific arousal level (i.e., shifts from synchronized to desynchronized EEG activity) do not necessarily accompany increased selective attention, especially when the level of arousal is consistently high (i.e., shows large amounts of desynchronized EEG activity) through- 
out the experiment. It is therefore unlikely that the results of the present experiment can be interpreted as reflecting shifts in general level of arousal as seen by Green and Arduini (1954) and Kemp and Kaada (1975).

The principle finding of this experiment was the demonstration of a correspondence between the attentive state of the animal and hippocampal theta activity. The results suggest that the hippocampus may perform an inhibitory function in selective attention experiments, in which the OCB has been shown to inhibit auditory input to the central nervous system at the peripheral stages in the afferent auditory pathways (Oatman \& Anderson, 1977). Therefore, the function of attention in sensory perception may be to initiate, within the hippocampus, a gating mechanism in which the evoked potentials of irrelevant sensory stimuli are inhibited through the action of the OCB.

\section{REFERENCES}

ADEY, W. R. Hippocampal states and functional relations with cortico-subcortical systems in attention and learning. In W. R. Adey \& T. Tokizane (Eds.), Progress in brain research: Structure and function of the limbic system. Amsterdam: Elsevier, 1967.

Arnolds, D. E. A. T., Lopes da Silva, F. H., Aitink, J. W., \& KAMP, A. Hippocampal EEG and behavior in dog. I. Hippocampal EEG correlates of gross motor behavior. Electroencephalography and Clinical Neurophysiology, 1979, 46, 552-570.

BENNETT, T. L. Hippocampal EEG correlates of behavior. Electroencephalography and Clinical Neurophysiology, 1970, 28, 17-23.

BenNetT, T. L. Hippocampal theta activity and behavior-A review. Communications in Behavioral Biology, 1971, 6, 37-48.

BENNETT, T. L. The electrical activity of the hippocampus and processes of attention. In R. L. Isaacson \& K. H. Pribram (Eds.), The hippocampus (Vol. 2): Neurophysiology and behavior. New York: Plenum, 1975.

Black, A. H., Young, G. A., \& Batenchuk, C. Avoidance training of hippocampal theta waves in flaxedilized dogs and its relation to skeletal movement. Journal of Comparative and Physiological Psychology, 1970, 70, 15-24.

Buño, W. G., Velluti, R., Handler, P., \& Garcia-Austt, E. Neural control of the cochlear input in the wakeful free guinea pig. Physiology \& Behavior, 1966, 1, 23-25.

Butler, D. H., Kamlet, A. S., \& Monty, R. A. A multipurpose analysis of variance FORTRAN IV computer program. Psychonomics Monograph Supplement, 1969, 2(16, Whole No. 32).

Buzsáki, G., Grastyán, E., Tveritskaya, I. N., \& Czopf, J. Hippocampal evoked potentials and EEG changes during classical conditioning in the rat. Electroencephalography and Clinical Neurophysiology, 1979, 47, 64-74.

Buzsáki, G., Haubenreiser, J., Grastyán, E., Czopf, J., \& KellénYi, L. Hippocampal slow wave activity during appetitive and aversive conditioning in the cat. Electroencephalography and Clinical Neurophysiology, 1981, 51, 276-290.

Coleman, J. R., \& Lindsley, D. B. Hippocampal electrical correlates of free behavior and behavior induced by stimulation of two hypothalamic-hippocampal systems in the cat. Experimental Neurology, 1975, 49, 506-528.
Coleman, J. R., \& Lindsley, D. B. Behavioral and hippocampal electrical changes during operant learning in cats and effects of stimulating two hypothalamic-hippocampal systems. Electroencephalography and Clinical Neurophysiology, 1977, 42, 309-331.

Douglas, R. J. The hippocampus and behavior. Psychological Bulletin, 1967, 67, 416-442.

Douglas, R. J. Pavlovian conditioning and the brain. In R. Boakes \& M. Halliday (Eds.), Inhibition and learning. London: Academic Press, 1972.

Doyle, J. C., Ornstein, R., \& Galin, D. Lateral specialization of cognitive mode: II. EEG frequency analysis. Psychophysiology, 1974, 567-578.

Elazar, A., \& Adey, W. R. Electroencephalographic correlates of learning in subcortical and cortical structures. Electroencephalography and Clinical Neurophysiology, 1967, 23, 225-240.

Feldman, S. Neurophysiological mechanisms modifying afferent hypothalamo-hippocampal conduction. Experimental Neurology, 1962, 5, 269-291.

Frederickson, C. J., Lenig, R. B., \& Frederickson, M. H. Hippocampal RSA in cats during cued and noncued delayed response performance. Behavioral and Neural Biology, 1980, 28, 383-391.

Gevins, A. S., Zeitlin, G. M., Yingling, C. D., Doyle, J. C., Dedon, M. F., Schaffer, R. E., Roumasset, J. T., \& YeAGER, C. L. EEG patterns during cognitive tasks. I. Methodology and analysis of complex behaviors. Electroencephalography and Clinical Neurophysiology, 1979, 47, 693-703.

Grastyán, E., Lissák, K., Madarász, I., \& Donhoffer, H. Hippocampal electrical activity during the development of conditioned reflexes. Electroencephalography and Clinical Neurophysiology, 1959, 11, 409-430.

Green, J. D., \& Arduini, A. A. Hippocampal electrical activity in arousal. Journal of Neurophysiology, 1954, 17, 533-557.

HARPE R, R. M. Frequency changes in hippocampal electrical activity during movement and tonic immobility. Physiology \& Behavior, 1971, 7, 55-58.

Hernández-PEón, R. Reticular mechanisms of sensory control. In W. A. Rosenblith (Ed.), Sensory communication. Cambridge, Mass: M.I.T. Press, 1961.

KeMP, I. R., \& KAADA, B. R. The relation of hippocampal theta activity to arousal, attentive behavior and somato-motor movements in unrestrained cats. Brain Research, 1975, 95, 323-342.

McFarland, W. L., Teitelbaum, H., \& Hedges, E. K. Relationship between hippocampal theta activity and running speed in the rat. Journal of Comparative and Physiological Psychology, 1975, 88, 324-328.

Moore, J. W. Information processing in space-time by the hippocampus. Physiological Psychology, 1979, 7, 224-232.

NAUTA, W. J. H. Hippocampal projections and related neural pathways to the midbrain in the cat. Brain, 1958, 81, 319-340.

OAtman, L. C. Role of visual attention on auditory evoked potentials in unanesthetized cats. Experimental Neurology, 1971, 32, 341-356.

OAtman, L. C. Effects of visual attention on the intensity of auditory evoked potentials. Experimental Neurology, 1976, 51, 41-53.

Oatman, L. C., \& Anderson, B. W. Effects of visual attention on tone burst evoked auditory potentials. Experimental Neurology, 1977, 57, 200-211.

Parmeggiani, P. L., \& Rapisarda, C. Hippocampal output and sensory mechanisms. Brain Research, 1969, 14, 387-400.

REDDING, F. K. Modification of sensory cortical evoked potentials by hippocampal stimulation. Electroencephalography and Clinical Neurophysiology, 1967, 22, 74-83.

Robinson, T. E., \& VANDERWOLF, C. H. Electrical stimulation of the brain stem in freely moving rats. II. Effects on hippocam- 
pal and neocortical electrical activity and relations to behavior. Experimental Neurology, 1978, 61, 485-515.

Solomon, P. R. Temporal versus spatial information processing theories of hippocampal function. Psychological Bulletin, 1979, 86, $1272-1279$.

Solomon, P. R. A time and a place for everything? Temporal processing views of hippocampal function with special reference to attention. Physiological Psychology, 1980, 8, 254-261.

Solomon, P. R., \& Moore, J. W. Latent inhibition and stimulus generalization of the classically conditioned nictitating membrane response in rabbits (Oryctolagus cuniculus) following dorsal hippocampal ablation. Journal of Comparative and Physiological Psychology, 1975, 89, 1192-1203.
VANDERWOLF, C. H. Limbic-diencephalic mechanisms of voluntary movement. Psychological Review, 1971, 78, 83-113.

Whishaw, I. Q., \& VANDE RWOLF, C. H. Hippocampal EEG and behavior: Effects of variation in body temperature and relation of EEG to vibrissae movement, swimming and shivering. Physiology \& Behavior, 1971, 6, 391-397.

Whishaw, I. Q., \& Vanderwolf, C. H. Hippocampal EEG and behavior: Changes in amplitude and frequency of RSA (theta rhythm) associated with spontaneous and learned movement patterns in rats and cats. Behavioral Biology, 1973, 8, 461-484.

(Manuscript received July 6, 1982;

revision accepted for publication August 20, 1982.) 